\title{
Описание осцилляций намагниченности кремниевой наноструктуры в слабых полях при комнатной температуре. Формула Лифшица-Косевича с переменной эффективной массой носителей
}

\author{
(C) В.В. Романов ${ }^{1}$, В.А. Кожевников ${ }^{1}$, В.А. Машков ${ }^{1}$, Н.T. Баграев ${ }^{1,2}$ \\ ${ }^{1}$ Санкт-Петербургский политехнический университет Петра Великого, \\ 195251 Санкт-Петербург, Россия \\ ${ }^{2}$ Физико-технический институт им. А.Ф. Иоффре Российской академии наук, \\ 194021 Санкт-Петербург, Россия \\ E-mail: bagraev@mail.ioffe.ru
}

Поступила в Редакцию 23 июля 2020 г.

В окончательной редакции 3 августа 2020 г.

Принята к публикации 3 августа 2020 г.

\begin{abstract}
Развит формализм статистического подхода к описанию осцилляций де Гааза-ван Альфена, известного как формула Лифшица-Косевича, применительно к низкоразмерной системе с зависящей от внешнего магнитного поля эффективной массой носителей. Статистический подход позволяет провести более детальную интерпретацию полученных в эксперименте результатов и провести анализ взаимосвязи обнаруженной нами зависимости эффективной массы носителя с индивидуальными особенностями структуры кремниевого наносандвича, обусловленными формированием в его составе negative-U $\delta$-барьеров.
\end{abstract}

Ключевые слова: формула Лифшица-Косевича, эффект де Гааза-ван Альфена, кремниевый наносандвич, эффективная масса, размерное квантование.

DOI: 10.21883/FTP.2020.12.50233.9493

\section{1. Введение}

Изучая магнитные свойства электронов в тонких слоях металлов в случае произвольного закона дисперсии, И.М. Лифшиц и А.М. Косевич, определив уровни энергии квазичастицы в магнитном поле и вычислив осциллирующую часть магнитного момента газа таких квазичастиц, получили общие формулы для исследования эффекта де Гааза-ван Альфена $[1,2]$. Следует отметить, что с развитием технологий получения низкоразмерных структур и регистрации на них осцилляций, идентифицированных как эффект де Гааза-ван Альфена, статистический подход, разработанный И.М. Лифшицем и А.М. Косевичем [1,2], не утратил своей значимости, позволяя в целом адекватно описать наблюдаемое явление. Тем не менее Шёнберг, скрупулезно рассмотрев факторы, влияющие на поведение намагниченности в тонких слоях металлов и в двумерных структурах, показал, что при описании эффекта де Гааза-ван Альфена формулой Лифшица-Косевича должны обсуждаться как аддитивные диамагнитный и парамагнитный вклады в намагниченность структуры, на фоне которых наблюдаются исследуемые осцилляции, так и характеристики самих осцилляций - их размах и форма [3]. Причем результат вычисления неосциллирующей составляющей намагниченности зависит от выбранного закона дисперсии, который определяет значение фазовой поправки $\gamma$ [3]. Непосредственный расчет фазовой поправки весьма проблематичен из-за того, что, как следует из работ $[3,4]$, ее значение при произвольном законе дисперсии может изменяться сложным образом во внеш- нем магнитном поле. Однако величина $\gamma$ может быть определена из эксперимента. Именно данная проблема, по-видимому, и привела к тому, что А.М. Косевич и И.М. Лифшиц отказались от детальных вычислений этой составляющей намагниченности [2].

В ставшем классикой выводе формулы ЛифшицаКосевича [1] предполагалось, что эффективная масса носителя как ключевой параметр вычислительной процедуры определяется только свойствами структуры, обеспечивающими экспериментальное наблюдение эффекта де Гааза-ван Альфена, и не изменяется под воздействием на исследуемую структуру внешних факторов, варьирующихся в процессе измерений, в частности, магнитного поля. В то же время Датта [5] обратил внимание на, что такие характеристики осцилляций де Гааза-ван Альфена, как эффективная масса носителя, могут зависеть от напряженности внешнего магнитного поля. Особое внимание данному вопросу было уделено в связи с развитием исследований квантового эффекта Холла и эффекта де Гааза-ван Альфена в низкоразмерных полупроводниковых структурах, в частности в гетероструктуре $\mathrm{GaAs} / \mathrm{AlGaAs}$, где осцилляции наблюдались при низких температурах и высоких магнитных полях [6-10].

Следует обратить внимание на то, что эксперименты по наблюдению осцилляций де Гааза-ван Альфена, выполненные на гетероструктурах $\mathrm{GaAs} / \mathrm{AlGaAs}$ требовали предварительного оптического воздействия на исследуемый образец, охлажденный до сверхнизких температур [6-10]. Причем обнаруженный эффект составлял 
$\sim 10^{-5}$ от наблюдаемого эффекта без предварительного оптического воздействия [10]. Здесь уместно заметить, что при регистрации квантового эффекта Холла дробная составляющая также наблюдалась только после предварительной подсветки [9].

В ряде работ [6-10], посвященных наблюдению эффекта де Гааза-ван Альфена, осцилляции намагниченности демонстрируют определенную закономерность, проявляющуюся в монотонном возрастании их амплитуды с ростом напряженности внешнего магнитного поля. В то же время из цитируемых работ следует, что базовая, неосциллирующая, составляющая обнаруживает существенные различия в своем поведении.

Так, в работах [6-9] осцилляции де Гааза-ван Альфена наблюдались на фоне постоянной, не зависящей от напряженности внешнего магнитного поля, составляющей намагниченности. Тогда как в работе [10] неосциллирующая компонента намагниченности сложным немонотонным образом изменяется с ростом магнитного поля, однако во всех случаях авторы не обсуждали данный эффект.

В дальнейшем такое поведение полевой зависимости в эффекте де Гааза-ван Альфена получило объяснение в рамках модели оптической перезарядки метастабильных $\mathrm{DX}$-центров с отрицательной корреляционной энергией (negative-U), которые ограничивают краевые каналы гетероструктур, ответственные за эффекты квантового транспорта носителей $[5,11]$. Причем наиболее важную роль в усилении квантовых эффектов играла оптическая накачка монохроматическим светом с энергией, соответствующей фото-диссоциации нейтральных DX-центров:

$$
2 \mathrm{DX}^{0}+h v \Rightarrow \mathrm{DX}^{-}+\mathrm{DX}^{+} \text {. }
$$

Таким образом, проведенная подсветка предварительно охлажденного образца стимулировала оптически индуцированную negative-U, так как исходное состояние DX-центров в образце при низких температурах, из-за их метастабильности, было однократно заряженным. Формирующиеся в процессе подсветки диполи, состоящие из $\mathrm{DX}$-центров, $\mathrm{DX}^{-}+\mathrm{DX}^{+}$, после ее выключения сохранялись, благодаря их метастабильным свойствам [11], в течение длительного времени.

Таким образом, производимая после охлаждения гетероструктуры до сверхнизких температур и предшествующая началу измерений подсветка является необходимым этапом подготовки эксперимента, так как negative-U дипольные центры, ограничивающие краевые каналы, способны нивелировать негативное влияние электронэлектронного взаимодействия, открывая новые перспективы исследования макроскопических квантовых эффектов $[5,6-10]$.

Усиление negative-U свойств точечных и протяженных дефектов может привести к спонтанному формированию дипольных центров, цепочки которых ограничивают краевые каналы низкоразмерных структур. В этом случае непосредственно дипольный центр представляет собой основное состояние дефекта, и какого-либо дополнительного воздействия не требуется для его стабилизации.
Более того, negative-U свойства цепочек, состоящих из дипольных центров, могут даже усиливаться при их взаимодействии с носителями, находящимися внутри краевых каналов [11], тем самым открывая возможности для наблюдения макроскопических квантовых эффектов при высоких температурах и в слабых магнитных полях $[11-14]$.

Negative-U цепочки, состоящие из тригональных дипольных центров бора, были обнаружены при исследовании кремниевых сандвич-структур, представляющих собой сверхузкие, 2 нм, квантовые ямы $p$-типа (Si-QW), ограниченные $\delta$-барьерами, сильно легированными бором, на поверхности $n$-Si(100). Изучение макроскопических квантовых эффектов в транспорте носителей в краевых каналах сандвич-структур, ограниченных negative-U дипольными центрами бора, позволило идентифицировать сильное подавление электронэлектронного взаимодействия и значительное уменьшение эффективной массы дырок в краевых каналах в слабых магнитных полях вследствие их взаимодействия с дипольными цепочками, которое поддерживает negative-U реакцию $[11,13,15-19]$.

Эффект де Гааза-ван Альфена, относящийся к наиболее прямым методам измерения эффективной массы носителей, позволяет, кроме того, выявить особенности поведения одной из основных характеристик носителей, применяя различные подходы к описанию полевых зависимостей намагниченности исследуемых материалов.

Результаты, полученные при измерениях намагниченности кремниевых сандвич-структур, демонстрирующих эффект де Гааза-ван Альфена при комнатной температуре и слабых магнитных полях [11-14], обнаруживают уменьшение размаха амплитуды осцилляций намагниченности с возрастанием напряженности приложенного магнитного поля, а неосциллирующая составляющая намагниченности в целом ведет себя аналогично наблюдаемой в работе [10].

Ранее, используя относительно простую термодинамическую модель, нам удалось добиться согласия с экспериментом, допустив зависимость эффективной массы носителей от напряженности внешнего магнитного поля [14].

В настоящей работе для описания экспериментальной полевой зависимости намагниченности кремниевого наносандвича мы развиваем основанный на принципах статистической физики формализм, разработанный И.М. Лифшицем и А.М Косевичем, применительно к системе с зависящей от магнитного поля эффективной массой носителя, как более информативный и позволяющий, вообще говоря, описать форму осцилляций, наблюдаемых в эффекте де Гааза-ван Альфена.

\section{2. Формула Лифшица-Косевича с переменной эффективной массой носителя}

Модифицируем полученную в ставшем классическим выводе Лифшица-Косевича [1,2] формулу, описываю- 
щую эффект де Газа-ван Альфена, допуская при этом зависимость эффективной массы носителя от внешнего магнитного поля.

Поскольку расчет свободной энергии электронного газа с произвольным законом дисперсии

$$
F=N \mu-k T \sum_{i=1}^{N} \ln \left\{1+\exp \left(\frac{\mu-E_{i}}{k T}\right)\right\}
$$

где $\mu$ - химический потенциал, а сумма берется по всем возможным состояниям с энергией $E_{i}$, в случае зависящей от напряженности магнитного поля эффективной массы носителя полностью аналогичен вычислительной процедуре для постоянной эффективной массы [1,2], запишем, не приводя промежуточных выкладок, выражение, определяющее осциллирующую часть свободной энергии системы:

$$
\begin{aligned}
F= & 2 k T \sum_{s=1}^{\infty}(-1)^{s}\left(\frac{e H}{2 \pi s c \hbar}\right)^{3 / 2} \frac{1}{\sinh \left(2 \pi^{2} s k T / \hbar \omega_{c}^{*}\right)} \\
& \times \frac{1}{\sqrt{A_{0}^{\prime \prime}}} \cos \left(\frac{s c \hbar}{e H} A_{0}-\frac{\pi}{4}\right) .
\end{aligned}
$$

Здесь $\omega_{c}^{*}=\frac{e H}{m^{*} c}-$ циклотронная частота.

Пусть $A\left(k_{z}\right)$ - площадь сечения поверхности Ферми в $k$-пространстве слоем $k_{z}$. Тогда $A_{0}$ и $A_{0}^{\prime \prime}$ - параметры разложения функции $A\left(k_{z}\right)$ в ряд в окрестности точки $k_{z}=k_{0}$, где $\frac{\partial A}{\partial z}=0$, т.е. $A=A_{0}+k^{2} A^{\prime}, k=k_{z}-k_{0}-$ площадь экстремального сечения.

Так как магнитный момент $M$ выражается через свободную энергию $F$ как $M=-\frac{\partial F}{\partial H}$, а нас интересует осциллирующая часть магнитного момента, то

$$
\begin{aligned}
M & =2 k T \sum_{s=1}^{\infty}(-1)^{s+1}\left(\frac{e}{2 \pi s c \hbar}\right)^{3 / 2} \frac{1}{\sqrt{a_{0}^{\prime \prime}}} \\
& \times \frac{\partial}{\partial H}\left(H^{3 / 2} \frac{1}{\sinh \left(2 \pi^{2} s k T / \hbar \omega_{c}^{*}\right)} \cos \left(\frac{s c \hbar}{e H} A_{0}-\frac{\pi}{4}\right)\right) .
\end{aligned}
$$

Так как $\frac{\partial \omega_{c}^{*}}{\partial H}=\frac{e}{c} \frac{1}{m_{0}^{*}} \frac{1-\alpha H^{2}}{\left(1+\alpha H^{2}\right)^{2}}$, получаем

$$
\begin{aligned}
& \frac{\partial}{\partial H}\left(H^{3 / 2} \frac{1}{\sinh \left(2 \pi^{2} s k T / \hbar \omega_{c}^{*}\right)} \cos \left(\frac{s c \hbar}{e H} A_{0}-\frac{\pi}{4}\right)\right) \\
= & H^{3 / 2} \frac{1}{\sinh \left(2 \pi^{2} s k T / \hbar \omega_{c}^{*}\right)} \cos \left(\frac{s c \hbar}{e H} A_{0}-\frac{\pi}{4}\right) \\
& \times\left[\frac{3}{2} \frac{1}{H}+\frac{2 \pi^{2} s k T e}{\hbar c m_{0}^{*}} \frac{1}{\omega_{c}^{* 2}} \frac{1-\alpha H^{2}}{\left(1+\alpha H^{2}\right)^{2}} \operatorname{coth}\left(2 \pi^{2} s k T / \hbar \omega_{c}^{*}\right)\right. \\
& \left.+\frac{s c \hbar}{e} A_{0} \frac{1}{H^{2}} \tan \left(\frac{s c \hbar}{e H} A_{0}-\frac{\pi}{4}\right)\right] .
\end{aligned}
$$

В результате осциллирующая часть магнитногом омента определяется выражением

$$
\begin{aligned}
M & =2 k T\left(\frac{e}{2 \pi c \hbar}\right)^{3 / 2} \frac{1}{\sqrt{A_{0}^{\prime \prime}}} \sum_{s=1}^{\infty} \frac{(-1)^{s+1}}{s^{3 / 2}} H^{3 / 2} \\
& \times \frac{1}{\sinh \left(2 \pi^{2} s k T / \hbar \omega_{c}^{*}\right)} \cos \left(\frac{s c h}{e H} A_{0}-\frac{\pi}{4}\right) \\
& \times\left[\frac{3}{2} \frac{1}{H}+\frac{2 \pi^{2} s k T e}{\hbar c m_{0}^{*}} \frac{1}{\omega_{c}^{* 2}} \frac{1-\alpha H^{2}}{\left(1+\alpha H^{2}\right)^{2}} \operatorname{coth}\left(2 \pi^{2} s k T / \hbar \omega_{c}^{*}\right)\right. \\
& \left.+\frac{s c \hbar}{e} A_{0} \frac{1}{H^{2}} \tan \left(\frac{s c \hbar}{e H} A_{0}-\frac{\pi}{4}\right)\right] .
\end{aligned}
$$

Значение $A_{0}$ можно найти из периода осцилляций намагниченности: $\frac{c \hbar}{e H} A_{0}=2 \pi(n+\gamma)$, где $\gamma-$ фазовая поправка.

Из выражения (3) можно получить оценку эффективной массы носителя. Для наблюдения эффекта де Гаазаван Альфена требуется, чтобы аргумент гиперболической функции $2 \pi^{2} s k T / \hbar \omega_{c}^{*}$ был хотя бы порядка единицы. Поэтому в полях, напряженностью $H \sim 10^{2}-10^{3} \mathrm{Oe}$ при комнатной температуре $(T=300 \mathrm{~K})$, осцилляции намагниченности могут наблюдаться, когда эффективная масса носителя имеет порядок величины $m^{*} \sim 10^{-5}-10^{-4} m_{e}, m_{e}-$ масса электрона. Полученная оценка хорошо коррелирует с измерениями эффективной массы дырок при наблюдении циклотронного резонанса $[18,19]$. Особо следует отметить согласие с результатами, полученными путем регистрации осцилляций Ааронова-Кашера, которые учитывают не только воздействие внешнего магнитного поля, но и генерацию квантов магнитного потока в процессе протекания тянущего тока исток-сток $[16,20]$.

\section{3. Эксперимент}

Исследования кремниевой сандвич-структуры с характеристиками, описанными выше (см. работы [11-13]), проводились путем измерения и анализа полевой зависимости намагниченности методом Фарадея и выполнялись на установке Faraday Balance, созданной на базе спектрометра MGD 312 FG, при комнатной температуре в магнитных полях, напряженностью до $800 \mathrm{Oe}$ Развертка магнитного поля осуществлялась в пошаговом режиме с заданным значением $\Delta H$, причем регистрация интенсивности взаимодействия с магнитным полем производилась при достижении образцом состояния термодинамического равновесия, что контролировалось автоматизированной системой управления измерениями с помощью специально разработанного программного обеспечения.

Измерения намагниченности кремниевой сандвичструктуры были проведены с шагом по полю в 1 Ое, при котором удается наблюдать разрешенные осцилляции в эффекте де Гааза-ван Альфена до значений фактора заполнения $v=12$ включительно. 
Важнейшей особенностью проведенного экспериментального исследования является то, что до начала измерений образец длительное время хранился в закрытом контейнере в воздушной среде при комнатной температуре и не подвергался предварительно какимлибо внешним воздействиям, которые могли нарушить состояние термодинамического равновесия.

\section{4. Обсуждение и выводы}

Анализ полевой зависимости намагниченности, полученной в эксперименте (см. рисунок), основывался на полученном в работе [14] выражении, определяющем вклад магнитного поля в изменение эффективной массы носителей:

$$
m^{*}=\left(1+\alpha H^{2}\right) m_{0}^{*},
$$

где $m_{0}^{*}$ - эффективная масса носителейя в отсутствие внешнего магнитного поля; $\alpha$ - параметр, отвечающий за скорость возрастания массы с напряженностью поля.

Численные эксперименты с учетом выражения (3) показали, что наилучшее согласие с результатами опыта (см. рисунок), а также данными, полученными при электрическом и оптическом детектировании циклотронного резонанса $[18,19]$, достигается при значениях констант: $m_{0}^{*}=6 \cdot 10^{-5} m_{e}$ и $\alpha=3 \cdot 10^{-6}$.

Проведенное описание экспериментальной зависимости построено на принципах статистической физики. Обобщение формулы Лифшица- Косевича $[1,2]$ с произвольным законом дисперсии выполнено для описания осцилляций де Газа-ван Альфена, наблюдаемых в структурах с зависящей от магнитного поля эффективной массой носителей. Вычисленная амплитуда осцилляций, как и следует из эксперимента, убывает с ростом напряженности внешнего магнитного поля. Полученная из

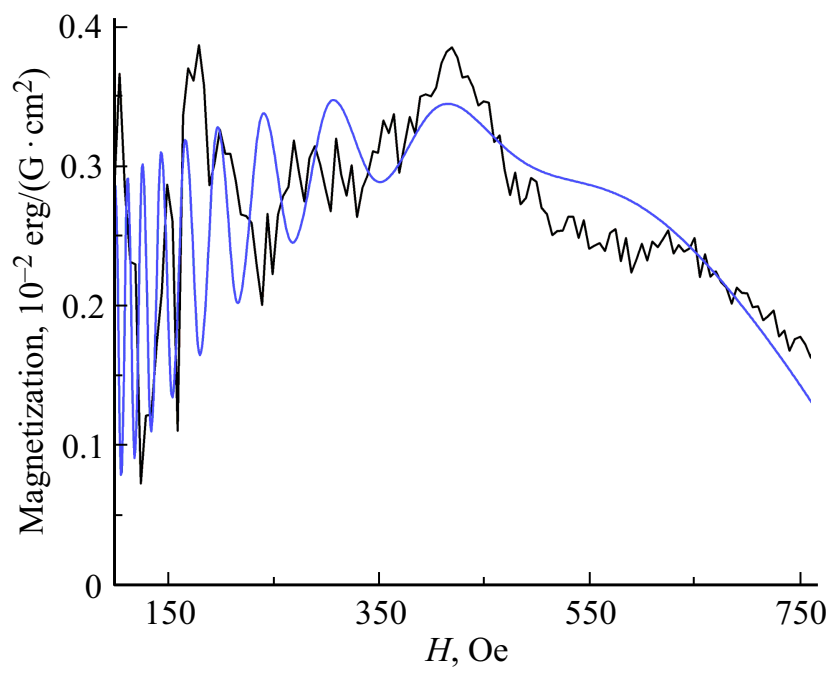

Полевая зависимость намагниченности кремниевого наносандвича, демонстрирующая эффект де Гааза-ван Альфена при комнатной температуре в слабых магнитных полях. Неосциллирующая составляющая намагниченности аппроксимирована параболическим законом. обобщенной формулы Лифшица-Косевича зависимость амплитуды осцилляций от напряженности внешнего магнитного поля согласуется с результатами работы [14], в которой использовалась сравнительно простая термодинамическая модель.

Вопреки существовавшему мнению $[1,2]$, непериодическая составляющая существенно влияет на форму полевой зависимости намагниченности, наблюдаемой в эксперименте (см. рисунок). Из приведенных исследований вытекает, что значение фазовой поправки $\gamma$ сложным образом зависит от напряженности приложенного магнитного поля и, по-видимому, должно определяться из данных эксперимента.

На рисунке изменение с магнитным полем неосциллирующей компоненты намагниченности кремниевого наносандвича аппроксимировано для наглядности параболическим законом, что, по-видимому, является достаточно грубым приближением и приводит к смещению по „высоте“ осцилляций при некоторых целочисленных факторах заполнения.

\section{5. Заключение}

Таким образом, полевая зависимость намагниченности кремниевого наносандвича при комнатной температуре в слабых магнитных полях получает адекватную интерпретацию при использовании обобщенной формулы Лифшица-Косевича, в которой учитывается зависимость эффективной массы носителей от внешнего магнитного поля.

Следует отметить, что полученная в работе [14] полевая зависимость эффективной массы носителей (4) указывает на возможную роль в ее формировании различных версий спин-поляронов, образующихся вследствие магнитоупорядочения дипольных negative-U оболочек через обменное взаимодействие с носителями в краевых каналах [21-24]. Таким образом, в слабых магнитных полях, по-видимому, доминирует спин-коррелированный перенос вдоль дипольных negative-U оболочек, в процессе которого стимулируется negative-U реакция, ответственная за подавление электрон-электронного взаимодействия и соответствующее уменьшение эффективной массы носителей [21-24]. С увеличением внешнего магнитного поля спин-коррелированный перенос разрушается и дипольные центры, ограничивающие краевые каналы, магнитоупорядочиваются посредством обменного взаимодействия [23,24], что сопровождается ростом эффективной массы носителей в канале и, соответственно, нивелированием макроскопических квантовых эффектов, в частности уменышением амплитуды осцилляций де Гааза-ван Альфена с возрастанием напряженности магнитного поля.

\section{Конфликт интересов}

Авторы заявляют, что у них нет конфликта интересов. 


\section{Список литературы}

[1] И.М. Лифшиц, А.М. Косевич. ЖЭТФ, 29, 730 (1955).

[2] А.М. Косевич, И.М. Лифшиц. ЖЭТФ, 29, 743 (1955).

[3] D. Shoenberg. Magnetic oscillations in metals. (Cambridge University Press, Cambridge, 1984).

[4] L.M. Roth. Phys. Rev., 145 (2), 434 (1966).

[5] Electronic Transport in Mesoscopic Systems. (Supriyo Datta, Cambridge University Press, 1995).

[6] I.D. Vagner, Tsofar Maniv, E. Ehrenfreund. Phys. Rev. Lett., 51 (18), 1700 (1983).

[7] J.G.E. Harris, R. Knobel, K.D. Maranowski, A.C. Gossard, N. Samarth, D.D. Awschalom. Phys. Rev. Lett., 86 (20), 4644 (2001).

[8] E. Gornik, R. Lassnig, G. Strasser, H.L. Stormer, A.C. Gossard, W. Wiegmann. Phys. Rev. Lett., 54, 1820 (1985).

[9] J.P. Eisenstein, H.L. Stormer, V. Narayanamurti, A.Y. Cho, A.C. Gossard, C.W. Tu. Phys. Rev. Lett., 55 (8), 875 (1985).

[10] M.P. Schwarz, M.A. Wilde, S. Groth, D. Grundler, Ch. Heyn, D. Heitmann. Phys. Rev. B, 65, 245315 (2002).

[11] N.T. Bagraev, V.Yu. Grigoryev, L.E. Klyachkin, A.M. Malyarenko, V.A. Mashkov, V.V. Romanov. Semiconductors, 50 (8), 1025 (2016).

[12] N.T. Bagraev, L.E. Klyachkin, V.V. Romanov, A.I. Ryskin. Low Temp. Phys., 40 (4), 352 (2014).

[13] N.T. Bagraev, V.Yu. Grigoryev, L.E. Klyachkin, A.M. Malyarenko, V.A. Mashkov, V.V. Romanov, N.I. Rul'. Low Temp. Phys., 43 (1), (2017).

[14] V.V. Romanov, V.A. Kozhevnikov, N.T. Bagraev, C.T. Tracey. Semiconductors, 53 (12), 1629 (2019).

[15] Н.Т. Баграев, А.Д. Буравлев, Л.Е. Клячкин, А.М. Маляренко, В. Гельхофф, В.К. Иванов, И.А. Шелых. ФТП, 36, 462 (2002).

[16] N.T. Bagraev, N.G. Galkin, W. Gehlhoff, L.E. Klyachkin, A.M. Malyarenko J. Phys.: Condens. Matter, 20, 164202-12 (2008).

[17] Н.Т. Баграев, Е.С. Брилинская, Д.С. Гец, Л.Е. Клячкин, А.М. Маляренко, В.В. Романов. ФТП, 45, 1503 (2011).

[18] Н.Т. Баграев, Р.В. Кузьмин, А.С. Гурин, Л.Е. Клячкин, А.М. Маляренко, В.А. Машков. ФТП, 48 (12), 1646 (2014).

[19] Н.Т. Баграев, Д.С. Гец, Э.Ю. Даниловский, Л.Е. Клячкин, А.М. Маляренко. ФТП, 47 (4), 503 (2013).

[20] N.T. Bagraev, V.S. Khromov, L.E. Klyachkin, A.M. Malyarenko. Abstract Int. Symp. Spin Waves, June 3-8, 2018 (St. Petersburg, Russia) p. 102. ISBN 978-5-00125-053-1 (2018)

[21] Н.Ф. Мотт. Переходы металл-изолятор (М., Наука, 1979) c. 171.

[22] Э.Л. Нагаев. Физика магнитных полупроводников (М., Наука, 1979) c. 225.

[23] Н.Т. Баграев, А.И. Гусаров, В.А. Машков. ЖЭТФ, 92, 968 (1987).

[24] Н.Т. Баграев, А.И. Гусаров, В.А. Машков. ЖЭТФ, 95, 1412 (1989).

Редактор А.Н. Смирнов

\section{The description of oscillations of the magnetization in silicon nanosandwich at room temperature in weak magnetic fields. Lifshitz-Kosevich expression with variable carrier effective mass}

\author{
V.V. Romanov ${ }^{1}$, V.A. Kozhevnikov ${ }^{1}$, V.A. Mashkov ${ }^{1}$, \\ N.T. Bagraev ${ }^{\mathbf{1 , 2}}$
}

${ }^{1}$ Peter the Great St. Petersburg Polytechnic University, 195251 St. Petersburg, Russia

2 loffe Institute,

194021 St. Petersburg, Russia

Abstract In the present work, a form of the statistical approach to the description of the de Haas-van Alphen oscillations is developed, known as the Lifshitz-Kosevich formula, as applied to a low-dimensional system with the carrier effective mass dependence on the magnetic field. The statistical approach allows a more detailed interpretation of the experimental results and analyze the relationship between the found by us dependence of the effective mass of the carrier with the individual characteristics of the silicon nanosandwich structures, which are determined by the formation of negative- $\mathrm{U} \delta$-barriers in its composition. 